\title{
Fungal spondylodiscitis in a patient recovered from H7N9 virus infection: a case study and a literature review of the differences between Candida and Aspergillus spondylodiscitis ${ }^{*}$
}

\author{
Lie-dao YU ${ }^{\S}$, Zhi-yun FENG ${ }^{\S}$, Xuan-wei WANG, Zhi-heng LING, Xiang-jin LIN ${ }^{\dagger \ddagger}$ \\ (Department of Orthopedic Surgery, the First Affiliated Hospital, School of Medicine, Zhejiang University, Hangzhou 310003, China) \\ †E-mail: doclinxiangjin@163.com \\ Received Feb. 24, 2016; Revision accepted June 23, 2016; Crosschecked Oct. 18, 2016
}

\begin{abstract}
To report a rare case of fungal spondylodiscitis in a patient recovered from H7N9 virus infection and perform a literature review of the different characteristics of Candida and Aspergillus spondylodiscitis, we reviewed cases of spondylodiscitis caused by Candida and Aspergillus species. Data, including patients' information, pathogenic species, treatment strategy, outcomes, and relapses, were collected and summarized. The characteristics of Candida and Aspergillus spondylodiscitis were compared to see if any differences in clinical features, management, or consequences could be detected. The subject of the case study was first misdiagnosed as having a vertebral tumor, and then, following open biopsy, was diagnosed as having fungal spondylodiscitis. The patient made a good recovery following radical debridement. Seventy-seven additional cases of Candida spondylodiscitis and 94 cases of Aspergillus spondylodiscitis were identified in the literature. Patients with Candida spondylodiscitis tended to have a better outcome than patients with Aspergillus spondylodiscitis (cure rate $92.3 \%$ vs. $70.2 \%$ ). Candida was found more frequently (47.8\%) than Aspergillus (26.7\%) in blood cultures, while neurological deficits were observed more often in patients with Aspergillus spondylodiscitis (43.6\% vs. $25.6 \%$ ). Candida spinal infections were more often treated by radical debridement $(60.5 \%$ vs. $39.6 \%)$. Patients with Candida spondylodiscitis have better outcomes, which may be associated with prompt recognition, radical surgical debridement, and azoles therapy. A good outcome can be expected in fungal spondylodiscitis with appropriate operations and anti-fungal drugs.
\end{abstract}

Key words: Spondylodiscitis, Spinal infection, H7N9 virus, Avian influenza virus, Candida, Aspergillus, Fungi, Review http://dx.doi.org/10.1631/jzus.B1600077

CLC number: R681.5+1

\section{Introduction}

Invasive fungal spinal infection is a rare condition, compared with pyogenic or tuberculous spondylodiscitis (Broner et al., 1996; Chia et al., 2005; D'Agostino et al., 2010). It often occurs as an op-

\footnotetext{
Corresponding author

$\S$ The two authors contributed equally to this work

* Project supported by the Zhejiang Medical Technology and Education Foundation (No. 2015107339), China

\# Electronic supplementary materials: The online version of this article (http://dx.doi.org/10.1631/jzus.B1600077) contains supplementary materials, which are available to authorized users

(10) ORCID: Xiang-jin LIN, http://orcid.org/0000-0003-0062-0448

CC Zhejiang University and Springer-Verlag Berlin Heidelberg 2016
}

portunistic infection in immunocompromised patients, but immunocompetents can also be affected. Back pain is the most frequent manifestation, but neurological deficits may sometimes occur (Kim et al., 2006). Diagnosis of fungal spondylodiscitis without a biopsy or operation is very difficult, due to the non-specific symptoms, the low rate of positive blood cultures, and atypical radiological findings.

The optimal management of fungal spondylodiscitis remains uncertain. Several reports suggest that fungal spondylodiscitis without neurological deficits and spinal instability can be treated merely by anti-fungal drugs (Sugar et al., 1990; Cortet et al., 1994; Hennequin et al., 1996; Rachapalli et al., 2010; 
Ersoy et al., 2011; Theodoros and Sotirios, 2012). Others have argued that surgical treatments should be given as soon as there is no clear diagnosis and when conventional treatment has failed, and should not be limited to cases with neurological presentations and spinal instabilities (Nasca and McElvein, 1985; Bridwell et al., 1990; Frazier et al., 2001; Hendrickx et al., 2001; Lenzi et al., 2004; Skaf et al., 2010; Iwata et al., 2014). Since a delayed operation may lead to a poor outcome (Iwata et al., 2014), many doctors recommend prompt surgical intervention. However, answers to some important questions about surgery for fungal spondylodiscitis are still unclear, such as how debridement should be given and how to choose drugs postoperatively.

As Aspergillus and Candida are reported to be two main etiologies for fungal spinal infections (Skaf et al., 2010), a literature review of spinal infections caused by Aspergillus and Candida may be helpful to clarify these questions. Also, there are no reports regarding the differences between the characteristics of Candida and Aspergillus spondylodiscitis. The virulence of Aspergillus has been shown to be stronger than that of Candida. Furthermore, Aspergillus infections in other systems have high mortality rates. It is unclear whether the consequences of Candida and Aspergillus spondylodiscitis are different, or if there should be any differences in their management. These are important issues, as they affect the decision making of doctors when confronted with fungal spondylodiscitis.

Here, we report a case of Candida spondylodiscitis in a patient recovered from H7N9 infection. We also review the characteristics of spondylodiscitis caused by Candida and Aspergillus described in previous reports, in the hope that this can provide insights for the management of fungal spinal infections.

\section{Case presentation}

In April 2013, a 76-year-old man, with a past medical history of H7N9 virus infection, presented with progressive lumbar pain for three months. On April 11, 2013, he had a heavy cough and was diagnosed at our hospital as having an H7N9 infection (Gao et al., 2013). The patient had severe pulmonary inflammation due to the H7N9 infection (Fig. 1). He was transferred to the intensive care unit, and antivirus and glucocorticoid treatments were given immediately (Gao et al., 2013). After one month, the patient showed a good clinical progression and was discharged. Three months later, he complained of pain in the lower back and left leg, accompanied by a low fever. The pain was unbearable and was aggravated by a change of position. Magnetic resonance imaging (MRI) showed signal changes at the L5 and S1 vertebral body, without disc involvement or any surrounding abscess (Figs. 2a-2c). Emission computed tomography indicated a concentration of radioactivity on the L5 vertebra. Therefore, a spinal tumor was first considered and a biopsy through the vertebral pedicle was performed. Pathological tests showed that the sample consisted of inflammatory fibroid tissue with active $\mathrm{T}$ lymphocyte proliferation, but there was no sign of tumor.

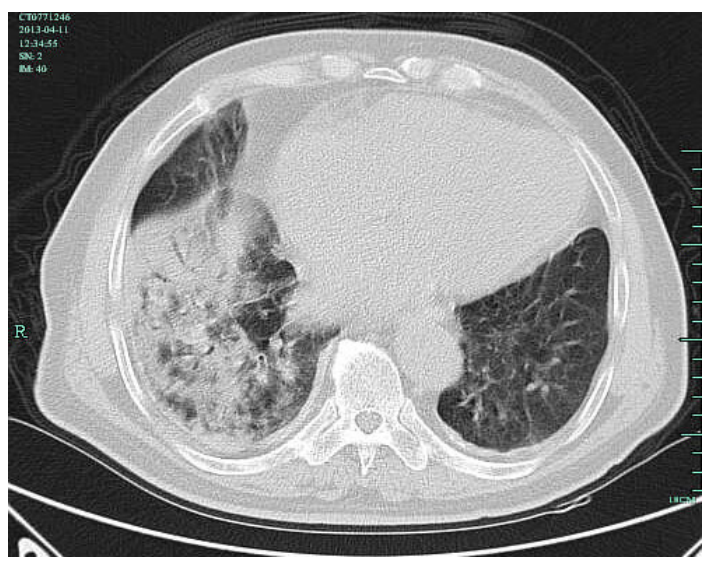

Fig. 1 Computed tomographic scan of the chest of the patient

After the onset of infection by H7N9, consolidations were observed in almost the whole right lung and ground-glass opacities were found in the left lung

During this period, little pain relief was achieved by pain killers, and the low fever continued. Another MRI scan revealed signal changes in the L5 and S1 endplate with the disc involved, indicating spondylodiscitis at the L5/S1 level (Figs. 2d-2f). At this time, lumbar spine tuberculosis (TB) was considered. The patient underwent conventional anti-TB therapy with isoniazid, rifampicin, ethambutol, and moxifoxacin. However, two months of chemotherapy for TB produced no improvement. Based on the limited response to anti-TB therapy, we realized that TB might not be the pathogen. 


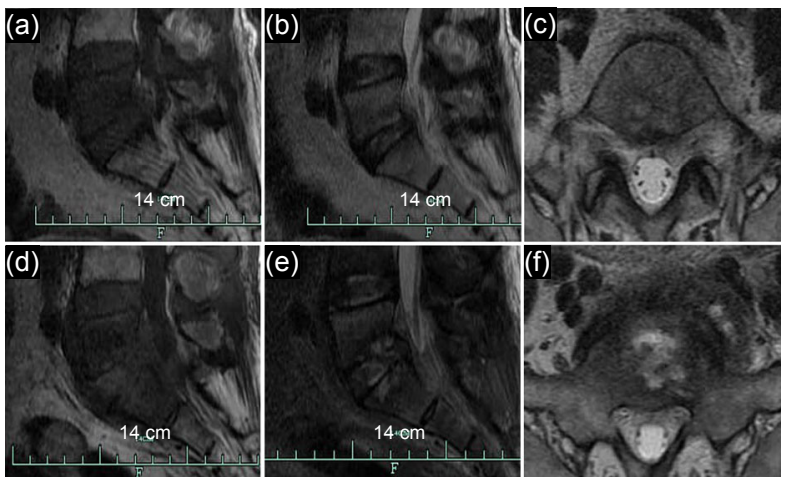

Fig. 2 Lumbar magnetic resonance images (MRIs) of the patient

MRI at the first time showed hypo-intensity on the whole L5 vertebrae body and the posterior endplate of S1 on the sagittal T1 weighted (T1W) image (a), while hyper-intensity can be observed only at the endplate of L5 on the sagittal T2 weighted (T2W) image (b). Mild signal changes can also be observed on the lateral image (c). Repeated MRI two months later showed that the area of hypo-intensity had widened, with the S1 vertebra body and the adjacent disc involved on the sagittal T1W image (d). Also, there were increased signal changes at the L5/S1 disc and adjacent endplates on the sagittal T2W image with both endplates having "moth-eaten" damage (e). The lateral $\mathrm{T} 2 \mathrm{~W}$ image showed erosion at the center of S1 vertebra (f)

The patient was then transferred to our department, as the pain was aggravated and accompanied by ache and numbness in the left lower limb. An exploration of the L5/S1 disc through the posterior medial approach was recommended to determine the source of the problem and also to perform a debridement. At surgery, the L5/S1 intervertebral disc and adjacent vertebral bodies appeared damaged. There was no abscess or pus, but a mass of granulation tissue was visible. The infected tissue with adjacent endplates was resected radically. An auto-bone graft and interbody fixation were inserted in the disc space. A direct smear and culture of the disc lesions showed no bacteria or mycotic organisms, but grew Candida albicans. Histologically, there was chronic inflammatory granuloma formation, necrosis, and fungal spores. Therefore, the patient received fluconazole intravenously for 1 month and then orally for 3 months. One year postoperatively, he was without back pain.

\section{Materials and methods}

PubMed and Google Scholar were searched using the terms spondylodiscitis, spondylitis, vertebral, diskitis, disc, fungal, fungi, Aspergillus, and Candida . The language was restricted to English, and the species to humans. No other limit was used. Cases of spondylodiscitis caused by Candida and Aspergillus species were selected. References cited by these reports were thoroughly screened to find additional cases. All the data, including basic information (age, gender, complaints, fever or not, blood culture findings, neurological deficits, infection sites), pathogenic species, treatment strategy, outcomes, and relapses were recorded if stated unambiguously.

Specimens had to be confirmed by pathological tests or cultures from infected tissues. Anti-fungal drugs before and after surgery were recorded separately. The treatment strategy was classified as antifungal only, anti-fungal followed by surgery, or surgery followed by anti-fungal. The surgical options were classified as simple debridement (including laminectomy and debridement without fusion) and radical debridement (radical debridement with fusion). The drug selections postoperatively were classified as amphotericin $\mathrm{B}(\mathrm{amB})$ only, azoles only, amB+ fluorocytosine, amB+azoles, amB+fluorocytosine + azoles, or micafungin/caspofungin.

\section{Results}

One hundred and seventy-one cases of Candida and Aspergillus spinal infections were identified in the literature (supplementary material). The characteristics of patients, including our patient, are summarized in Table 1. The mean age was 52.8 years (range, 1189 years). Most of the patients were men (70.9\%).

\subsection{Pathogenesis}

Among the 172 cases, 78 patients (45.3\%) had documented Candida spinal infection, while 94 (54.7\%) had Aspergillus spondylodiscitis (Table 1). The fungi were not commonly found in blood samples: only $55(32.0 \%)$ cases showed positive findings, $41.0 \%$ of Candida spondylodiscitis and $24.5 \%$ of Aspergillus spondylodiscitis cases (Table 1).

\subsection{Clinical presentation}

Back pain was the main complaint associated with fungal spinal infection. Almost all the patients suffered from back pain to variable degrees. Fever was not commonly seen $(31.4 \%)$, with $35.9 \%$ in 
Candida spondylodiscitis and $27.7 \%$ in Aspergillus spondylodiscitis cases (Table 1). Neurological deficits were observed in 61 patients $(35.5 \%)$, and were present more often in Aspergillus spondylodiscitis (43.6\% vs. $25.6 \%$ ) (Table 1). In 109 patients (63.4\%), lumbar vertebrae were solely involved, and in 42 patients $(24.4 \%)$, the thoracic spine was solely involved. The cervical spine was solely involved in only 2 patients with Candida spondylodiscitis. Multiple spinal infections were often caused by Aspergillus, with lumbar and thoracic spinal regions being the main concomitant sites (14 cases, 8.1\%) (Table 1).

Table 1 Characteristics of patients with Aspergillus and Candida spinal infections

\begin{tabular}{lcc}
\hline \multicolumn{1}{c}{ Characteristics } & Candida $(n=78)$ & Aspergillus $(n=94)$ \\
\hline Age & $55.2 \pm 18.0$ & $46.4 \pm 15.1$ \\
Gender & & \\
Male & $58(74.4 \%)$ & $64(68.1 \%)$ \\
Location & & \\
$\mathrm{C}$ & $2(2.6 \%)$ & 0 \\
$\mathrm{~T}$ & $20(25.6 \%)$ & $22(23.4 \%)$ \\
$\mathrm{L}$ & $51(65.4 \%)$ & $58(61.7 \%)$ \\
$\mathrm{C}+\mathrm{T}$ & 0 & $3(3.2 \%)$ \\
$\mathrm{T}+\mathrm{L}$ & $5(6.4 \%)$ & $9(9.6 \%)$ \\
$\mathrm{C}+\mathrm{T}+\mathrm{L}$ & 0 & $2(2.1 \%)$ \\
Fever & $28(35.9 \%)$ & $26(27.7 \%)$ \\
Pain & $78(100 \%)$ & $94(100 \%)$ \\
Neurological deficit & $20(25.6 \%)$ & $41(43.6 \%)$ \\
Blood culture & $32(41.0 \%)$ & $23(24.5 \%)$ \\
Operation $^{\mathrm{a}}$ & $44(56.4 \%)$ & $65(69.1 \%)$ \\
Outcome $^{\mathrm{b}}$ & $72(92.3 \%)$ & $66(70.2 \%)$ \\
Relapse & $7(9.0 \%)$ & $8(8.5 \%)$ \\
\hline
\end{tabular}

C: cervical spine; $\mathrm{T}$ : thoracic spine; L: lumbar spine. ${ }^{\mathrm{a}} 31$ cases did not refer to data of blood culture (17 for Candida and 14 for Aspergillus); ${ }^{\mathrm{b}} 6$ cases did not have data of outcome (each three for Candida and Aspergillus). Data were present as number of cases (percentage)

\subsection{Treatments}

Sixty-two of the 172 patients $(36.0 \%)$ received only drug therapy, $54(31.4 \%)$ required an operation after the failure of drug therapy, and 56 (32.6\%) had an early operation combined with anti-fungal drugs (Table 2).

As for the surgical plan, 47 patients received simple debridement or laminectomy. The other 44 patients were treated with radical excision and bone grafts. For 19 patients, no surgical information was available (Table 3 ).
Table 2 Number of patients with Candida and Aspergillus spondylodiscitis receiving conservative or operative treatment

\begin{tabular}{lcc}
\hline Treatment strategy & Candida & Aspergillus \\
\hline $\begin{array}{c}\text { Conservative treatment } \\
\text { Anti-fungals only }\end{array}$ & 34 & 28 \\
$\begin{array}{l}\text { Operative treatment } \\
\text { Anti-fungals failed, } \\
\text { then operation }\end{array}$ & 20 & 35 \\
$\begin{array}{l}\text { Operation directly, then } \\
\text { anti-fungals }\end{array}$ & 24 & 31 \\
\hline
\end{tabular}

Table 3 Surgical options for Candida and Aspergillus spondylodiscitis

\begin{tabular}{lcc}
\hline \multicolumn{1}{c}{ Surgical strategy } & Candida & Aspergillus \\
\hline Simply debridement $^{\mathrm{a}}$ & 15 & 32 \\
Radical debridement $^{\mathrm{a}}$ & 23 & 21 \\
Approach (A:P:A+P) & $13: 17: 0$ & $14: 34: 3$ \\
Stage $(1: 2)$ & $30: 1$ & $48: 4$ \\
\hline${ }^{\mathrm{a}}$ Twelve cases did not refer to the detailed information about the \\
operation way (6 for Candida, 13 for Aspergillus). ${ }^{\mathrm{b}}$ Twenty- \\
nine cases did not refer to the approach of operations (14 for \\
Candida and 15 for Aspergillus). In addition, transthoracic and \\
extrapleural anterolateral approaches were classified as anterior \\
approach, and costotransversectomy was classified as posterior \\
approach for surgery in the thoracic spine. A, anterior; P, pos- \\
terior; A+P, anterior plus posterior
\end{tabular}

Post-operative treatment information was available for 97 of the 110 patients who underwent surgery. Azoles were more likely to be used by patients with Candida spinal infection (19/40 vs. 19/57), and $\mathrm{amB}$ by patients with Aspergillus spinal infection (25/57 vs. $7 / 40)$ (Table 4). Combinations of $\mathrm{amB} /$ azoles (16/97), amB/fluorocytosine (5/97), and amB/ fluorocytosine/azoles (3/97) were not usually used after the surgery. New drugs, such as micafungin and caspofungin, were not commonly used after surgery: only three patients infected by Candida were given micafungin.

Table 4 Drug selections after operations

\begin{tabular}{lcc}
\hline \multicolumn{1}{c}{ Drug } & Candida & Aspergillus \\
\hline AmB only & 7 & 25 \\
AmB+fluorocytosine & 2 & 3 \\
Azoles only & 19 & 19 \\
AmB+azoles & 7 & 9 \\
AmB+fluorocytosine+azole & 2 & 1 \\
Micafungin/caspofungin & 3 & 0 \\
\hline
\end{tabular}

Azole includes voriconazole, fluconazole, itraconazole, miconazole, ketoconazole, cotrimoxazole, and posaconazole. AmB indicates amphotericin B. Four cases in Candida spinal infection and 9 cases in Aspergillus spinal infection have no data about the drug therapy post-operatively 


\subsection{Outcomes}

Outcomes could not be evaluated for six patients because of insufficient information. With the exception of two patients, all patients with Candida spinal infections were ultimately cured by means of medicine and/or surgical treatment. The cure rate for Candida spondylodiscitis (92.3\%) was higher than that for Aspergillus spondylodiscitis (70.2\%) (Table 1). Fifteen patients $(8.7 \%)$ suffered relapses. The relapse rate of Candida spondylodiscitis $(9.0 \%)$ paralleled that of Aspergillus spondylodiscitis (8.5\%) (Table 1).

\section{Discussion}

The pathogenic organisms of spondylodiscitis are often bacteria or Mycobacterium tuberculosis, while fungi are seldom implicated. However, the incidence of fungal spinal infections has increased in recent years, associated with the widespread use of broad-spectrum antibiotics, prolonged chemotherapeutic drugs, and corticosteroids (Kim et al., 2006; Shi et al., 2015). To our knowledge, this is the first recorded case of fungal spondylodiscitis found in a patient recovered from $\mathrm{H} 7 \mathrm{~N} 9$ infection. As outbreaks of H7N9 and other avian influenza virus infections increase worldwide (Liem et al., 2009; Yang et al., 2012; Chen et al., 2013; Gao et al., 2013; Uyeki and Cox, 2013), we should keep in mind that patients infected by such viruses may suffer from fungal spondylodiscitis, even after recovery from the viral infection.

Heavy corticosteroid consumption is the main risk factor for these opportunistic infections. Also, the viral infection may facilitate the proliferation of other pathogens growing in the blood, which may then seed in the vertebral body (Ceroni et al., 2013; 2014). Previous studies have shown that early viral infection can damage the mucosal layer of the airway, leading to respiratory tract bacterial infections (Yagupsky, 2004). A limitation of our study was that we did not check for H7N9 viral infection in the disc by polymerase chain reaction (PCR) or other assays to determine whether H7N9 might be involved in the pathogenesis of Candida spondylodiscitis. A deficiency of the immune system may be another factor predisposing for the invasion of Candida in the vertebral body. It is well accepted that viral infection, corticosteroid consumption, and prolonged periods of intensive care will cause temporary depression of the immune function, making patients susceptible to various types of infections.

Aspergillus and Candida have been shown to be the predominant causative fungi of spinal infections (Kim et al., 2006; Skaf et al., 2010), consistent with the findings of our review. Other species of fungi, such as Blastomyces, Pseudallescheria boydii, and Scremonium, are only rarely pathogens of spinal infections (Groll and Walsh, 2001; Kim et al., 2006; Skaf et al., 2010). In our study, the cure rate of Candida spinal infection was higher than that of Aspergillus (92.3\% vs. $70.2 \%$ ). This may be attributable to the difference in virulence between these pathogens. Systematic Aspergillus infection is life-threatening and has a high mortality rate (Mawk et al., 1983; Walsh, 2008; Pappas et al., 2009). Multiple vertebral infections by Aspergillus, as demonstrated by our review and Kwon et al. (2011), may also contribute to the lower cure rate of Aspergillus spinal infections. Early recognition of Candida spinal infection may also affect the different outcomes, since we found that patients with Candida spondylodiscitis had a higher positive rate of blood cultures. A more sensitive test is needed for detecting Aspergillus in blood cultures, because blood cultures are always the first step in testing patients with suspected infections.

In this review, only $36.0 \%$ of patients were treated by antifungal drugs alone. Such treatment often fails to stop the progression of bone destruction and can necessitate subsequent surgical intervention. Surgery was required following antifungal treatment in $32.0 \%$ of cases in this review and $30.0 \%$ of cases in another study (Hendrickx et al., 2001). The majority of patients were treated with surgical debridement, either to remove infected tissue, or to enable a definitive diagnosis, while indications for surgery varied from reports. Generally, open surgery could be divided into simple debridement and radical debridement with internal fixation. In this review, patients with Candida spondylodiscitis often underwent radical debridement, while simple debridement was chosen more frequently by patients with Aspergillus spondylodiscitis. Radical debridement can relieve the burden of infection and enhance the infiltration of antifungal drugs into the infected vertebra and disc. As Aspergillus spinal infection has a higher rate of 
mortality, a radical excision of the infected tissue is recommended if the condition of the patient permits. If not, patients with minimal bone destruction and a small abscess can be treated by endoscopic surgery in the early phase, without causing progressive instability (Iwata et al., 2014).

Even after surgical removal of infected tissue, sufficient anti-fungal treatment is very important. We collected information about drug selection postoperatively in the case reports to determine implications for clinical practice. AmB and azoles, used solely or in combination, were the basic regimens for the treatment of fungal spondylodiscitis. New drugs, such as caspofungin and micafungin, were always used when azole-resistance or amB-resistance occurred (Pemán et al., 2006; Theodoros and Sotirios, 2012; Tan et al., 2014). As the course of treatment varied from 1 to 24 months, these data were not analyzed. In general, amB was given for 2-8 weeks depending on the tolerance and response of patients, while azoles were always given for 3-24 months.

The key factor differentiating spondylodiscitis from a vertebral tumor in MRIs is the involvement of the disc, as the pathogens invade the endplates leading to infection of the disc. The initial MRIs in our case, however, showed no signal changes in the adjacent disc, consistent with the MR findings of fungal spondylodiscitis in previous studies (Williams et al., 1999; Shashidhar et al., 2014; Storm et al., 2014). This explains why our case was at first misdiagnosed as a vertebral tumor. Fungal spinal infections in the early phase are insidious, and MRI scans can mimic the presentation of a vertebral tumor.

The insidious clinical presentations, low frequency of positive blood cultures, non-specific radiological findings and poor awareness add to the difficulty in diagnosing fungal spinal infection (Frazier et al., 2001; Kim et al., 2006; Skaf et al., 2010). Biopsy or open surgery should be performed immediately when anti-bacterial or anti-TB treatments fail to control the symptoms. However, biopsy sometimes cannot provide an accurate answer (Palmisano et al., 2011; Shashidhar et al., 2014). A non-diagnostic aspirate should prompt an open biopsy for pathogen cultures and pathological examinations. Culture samples should be evaluated for not only bacteria and mycobacterial organisms but also fungi, if spondylodiscitis are suspected.
In conclusion, we should keep in mind that fungal spondylodiscitis can be a complication in patients with avian influenza virus infection. Candida spondylodiscitis tends to have a better outcome than Aspergillus spondylodiscitis, maybe due to earlier detection, radical debridement, and lengthy azoles treatment. With regard to treatment, the present case had a good result following one-stage radical debridement and internal fixation.

\section{Compliance with ethic guideline}

Lie-dao YU, Zhi-yun FENG, Xuan-wei WANG, Zhi-heng LING, and Xiang-jin LIN declared that they have no conflict of interest.

All procedures followed were in accordance with the ethical standards of the responsible committee on human experimentation (institutional and national) and with the Helsinki Declaration of 1975, as revised in 2008 (5). Informed consent was obtained from the patient for being included in the study and the work was approved by the ethical committee of the First Affiliated Hospital, School of Medicine, Zhejiang University (Hangzhou, China).

\section{References}

Bridwell, K., Campbell, J.W., Barenkamp, S.J., 1990. Surgical treatment of hematogenous vertebral Aspergillus osteomyelitis. Spine, 15(4):281-285. http://dx.doi.org/10.1097/00007632-199004000-00006

Broner, F.A., Garland, D.E., Zigler, J.E., 1996. Spinal infections in the immunocompromised host. Orthop. Clin. N. Am., 27(1):37-46.

Ceroni, D., Dubois-Ferrière, V., Cherkaoui, A., et al., 2013. 30 years of study of Kingella kingae: post tenebras, lux. Future Microbiol., 8(2):233-245. http://dx.doi.org/10.2217/fmb.12.144

Ceroni, D., Kampouroglou, G., Valaikaite, R., et al., 2014. Osteoarticular infections in young children: what has changed over the last years? Swiss Med. Wkly., 144: w13971.

Chen, Y., Liang, W.F., Yang, S.G., et al., 2013. Human infections with the emerging avian influenza A H7N9 virus from wet market poultry: clinical analysis and characterisation of viral genome. Lancet, 381(9881):1916-1925. http://dx.doi.org/10.1016/S0140-6736(13)60903-4

Chia, S.L., Tan, B.H., Tan, C.T., et al., 2005. Candida spondylodiscitis and epidural abscess: management with shorter courses of anti-fungal therapy in combination with surgical debridement. J. Infect., 51(1):17-23. http://dx.doi.org/10.1016/j.jinf.2004.08.020

Cortet, B., Richard, R., Deprez, X., et al., 1994. Aspergillus spondylodiscitis: successful conservative treatment in 9 cases. J. Rheumatol., 21(7):1287-1291.

D'Agostino, C., Scorzolini, L., Massetti, A.P., et al., 2010. A seven-year prospective study on spondylodiscitis: 
epidemiological and microbiological features. Infection, 38(2):102-107.

http://dx.doi.org/10.1007/s15010-009-9340-8

Ersoy, A., Dizdar, O.S., Koc, A.O., 2011. Aspergillus fumigatus spondylodiskitis in renal transplant patient: voriconazole experience. Exp. Clin. Transplant., 9(4): 265-269.

Frazier, D.D., Campbell, D.R., Garvey, T.A., et al., 2001. Fungal infections of the spine. Report of eleven patients with long-term follow-up. J. Bone Joint Surg. Am., 83-A(4):560-565.

Gao, H.N., Lu, H.Z., Cao, B., et al., 2013. Clinical findings in 111 cases of influenza A (H7N9) virus infection. N. Engl. J. Med., 368(24):2277-2285. http://dx.doi.org/10.1056/NEJMoa1305584

Groll, A.H., Walsh, T.J., 2001. Uncommon opportunistic fungi: new nosocomial threats. Clin. Microbiol. Infect., 7(Suppl. 2): 8-24. http://dx.doi.org/10.1111/j.1469-0691.2001.tb00005.x

Hendrickx, L., Wijngaerden, E.V., Samson, I., et al., 2001. Candidal vertebral osteomyelitis: report of 6 patients, and a review. Clin. Infect. Dis., 32(4):527-533. http://dx.doi.org/10.1086/318714

Hennequin, C., Bourée, P., Hiesse, C., et al., 1996. Spondylodiskitis due to Candida albicans: report of two patients who were successfully treated with fluconazole and review of the literature. Clin. Infect. Dis., 23(1):176-178. http://dx.doi.org/10.1093/clinids/23.1.176

Iwata, A., Ito, M., Abumi, K., et al., 2014. Fungal spinal infection treated with percutaneous posterolateral endoscopic surgery. J. Neurol. Surg. Part A: Cent. Eur. Neurosurg., 75(3):170-176. http://dx.doi.org/10.1055/s-0032-1329268

Kim, C.W., Perry, A., Currier, B., et al., 2006. Fungal infections of the spine. Clin. Orthop. Relat. Res., 444:92-99. http://dx.doi.org/10.1097/01.blo.0000203451.36522.4c

Kwon, J.W., Hong, S.H., Choi, S.H., et al., 2011. MRI findings of Aspergillus spondylitis. Am. J. Roentgenol., 197(5): W919-W923. http://dx.doi.org/10.2214/AJR.11.6786

Lenzi, J., Agrillo, A., Santoro, A., et al., 2004. Postoperative spondylodiscitis from Aspergillus fumigatus in immunocompetent subjects. J. Neurosurg. Sci., 48(2):81-85.

Liem, N.T., Tung, C.V., Hien, N.D., 2009. Clinical features of human influenza A (H5N1) infection in Vietnam: 2004 2006. Clin. Infect. Dis., 48(12):1639-1646. http://dx.doi.org/10.1086/599031

Mawk, J.R., Erickson, D.L., Chou, S.N., et al., 1983. Aspergillus infections of the lumbar disc spaces. Report of three cases. J. Neurosurg., 58(2):270-274. http://dx.doi.org/10.3171/jns.1983.58.2.0270

Nasca, R.J., McElvein, R.B., 1985. Aspergillus fumigatus osteomyelitis of the thoracic spine treated by excision and interbody fusion. Spine, 10(9):848-850. http://dx.doi.org/10.1097/00007632-198511000-00013

Palmisano, A., Benecchi, M., Filippo, M.D., 2011. Candida sake as the causative agent of spondylodiscitis in a hemodialysis patient. Spine J., 11(3):e12-e16.

http://dx.doi.org/10.1016/j.spinee.2011.01.026

Pappas, P.G., Kauffman, C.A., David, A., et al., 2009. Clinical practice guidelines for the management of candidiasis: 2009 update by the Infectious Diseases Society of America. Clin. Infect. Dis., 48(5):503-535. http://dx.doi.org/10.1086/596757

Pemán, J., Jarque, I., Bosch, M., et al., 2006. Spondylodiscitis caused by Candida krusei: case report and susceptibility patterns. J. Clin. Microbiol., 44(5):1912-1914. http://dx.doi.org/10.1128/JCM.44.5.1912-1914.2006

Rachapalli, S.M., Malaiya, R., Mohd, T., et al., 2010. Successful treatment of Candida discitis with 5-flucytosine and fluconazole. Rheumatol. Int., 30(11):1543-1544. http://dx.doi.org/10.1007/s00296-009-1215-x

Shashidhar, N., Tripathy, S.K., Balasubramanian, S., et al., 2014. Aspergillus spondylodiscitis in an immunocompetent patient following spinal anesthesia. Orthop. Surg., 6(1):72-77. http://dx.doi.org/10.1111/os.12091

Shi, J.M., Pei, X.Y., Luo, Y., et al., 2015. Invasive fungal infection in allogeneic hematopoietic stem cell transplant recipients: single center experiences of 12 years. $J$. Zhejiang Univ.-Sci. B (Biomed. \& Biotechnol.), 16(9): 796-804. http://dx.doi.org/10.1631/jzus.B1500005

Skaf, G.S., Kanafani, Z.A., Araj, G.F., et al., 2010. Non-pyogenic infections of the spine. Int. J. Antimicrob. Agents, 36(2):99-105. http://dx.doi.org/10.1016/j.ijantimicag.2010.03.023

Storm, L., Lausch, K.R., Arendrup, M.C., et al., 2014. Vertebral infection with Candida albicans failing caspofungin and fluconazole combination therapy but successfully treated with high dose liposomal amphotericin B and flucytosine. Med. Mycol. Case Rep., 6:6-9. http://dx.doi.org/10.1016/j.mmcr.2014.07.001

Sugar, A.M., Saunders, C., Diamond, R.D., 1990. Successful treatment of Candida osteomyelitis with fluconazole. A noncomparative study of two patients. Diagn. Microbiol. Infect. Dis., 13(6):517-520.

Tan, A.C., Parker, N., Arnold, M., 2014. Candida glabrata vertebral osteomyelitis in an immunosuppressed patient. Int. J. Rheuma. Dis., 17(2):229-231.

Theodoros, K., Sotirios, T., 2012. Successful treatment of azole-resistant Candida spondylodiscitis with high-dose caspofungin monotherapy. Rheumatol. Int., 32(9):29572958. http://dx.doi.org/10.1007/s00296-011-2121-6

Uyeki, T.M., Cox, N.J., 2013. Global concerns regarding novel influenza A (H7N9) virus infections. N. Engl. J. Med., 368(20): 1862-1864.

Walsh, T.J., 2008. Treatment of aspergillosis: clinical practice guidelines of the Infectious Diseases Society of America. Clin. Infect. Dis., 46(3):327-360. http://dx.doi.org/10.1086/525258 
Williams, R.L., Fukui, M.B., Meltzer, C.C., et al., 1999. Fungal spinal osteomyelitis in the immunocompromised patient: MR findings in three cases. Am. J. Neuroradiol., 20(20):381-385.

Yagupsky, P., 2004. Kingella kingae: from medical rarity to an emerging paediatric pathogen. Lancet Infect. Dis., 4(6): 358-367. http://dx.doi.org/10.1016/S1473-3099(04)01046-1

Yang, S.G., Cao, B., Liang, L.R., et al., 2012. Antiviral therapy and outcomes of patients with pneumonia caused by influenza A pandemic (H1N1) virus. PLOS ONE, 7(1):e29652. http://dx.doi.org/10.1371/journal.pone.0029652

\section{List of electronic supplementary materials}

Case reports or case serials related to the Candida or Aspergillus spondylosidcitis

\section{中文概要}

题 目: 念珠菌脊柱感染发生在 H7N9 治愈的患者一例报 道及文献分析念珠菌脊柱感染和曲霉菌脊柱感 染的临床区别
目 的: 报道在 H7N9 治愈患者中发现念珠菌脊柱感染病 例, 并总结念珠菌和曲需菌脊柱感染的临床表 现，处理方法和预后等不同点。

创新点：首次报道在 H7N9 离流感治愈患者中出现念珠菌 脊柱感染, 经过彻底的清创融合内固定和唑类抗 真菌药治疗取得良好疗效。文献研究发现了念珠 菌和曲霉菌这两大主要真菌在脊柱感染中的临 床表现、处理方法和临床结局均有不同。念珠菌 脊柱感染治愈率为 $92.3 \%$, 而曲霉菌为 $70.2 \%$ 。 其可能的原因有以下四点, 念珠菌血培养结果阳 性率更高（ $41.0 \%$ vs. $24.5 \%$ ) , 神经损害发生概率 更低（25.6\% vs. $43.6 \%$ ），手术方式更多地选用 彻底病灶清除加融合内固定 (60.5\% vs. 39.6\%), 术后多选用唑类抗真菌药。

方 法: 在 PubMed 和 Google Scholar 搜集念珠菌和曲霉 菌脊柱感染的病例, 提取患者一般信息、临床症 状、感染部位、病原学结果、治疗方法、治疗结 局和复发等情况进行总结分析。

结 论: 念珠菌脊柱感染的治愈率相对曲霉菌脊柱感染更 高, 这可能与念珠菌可以早期发现, 神经损害较 少, 更多地选用彻底病灶清除和唑类抗真菌药有 关。真菌脊柱感染可以通过合适的手术和抗真菌 药取得良好临床疗效。

关键词：真菌脊柱感染; H7N9 感染; 念珠菌; 曲霉菌; 综述 